\title{
SiOx coated plastic fiber optic sensor for gas monitoring in RPC
}

\section{S. Grassini*}

Dipartimento di Scienza Applicata e Tecnologia, Politecnico di Torino

E-mail: sabrina.grassiniepolito.it

\section{Ishtaiwi, M. Parvis}

Dipartimento di Elettronica e Telecomunicazioni, Politecnico di Torino

E-mail: maen.ishtaiwiepolito.it

\section{Benussi, S. Bianco, S. Colafranceschi, D. Piccolo}

Laboratori Nazionali INFN, Frascati

E-mail: stefano.Biancodnf.infn.it

This paper describes the development of a sensor designed to monitor the closed loop recirculation gas system of the Resistive Plate Counter (RPC) muon detectors which are used for the Compact Muon Solenoid (CMS) experiment installed at the Large Hadron Collider (LHC) accelerator of the European Center for Nuclear Research (CERN). The sensor is designed to detect low concentrations of hydrogen fluoride $(H F)$ in the gas mixtures and employs a plastic optic fiber (POF) which is covered with a thin layer of a glass-like material. The fluoride ions attacks the glass and alters the fiber transmission capability so that the detection simply requires a LED and a photodiode. The coating is obtained by means of a low pressure plasma enhanced chemical vapor deposition (PECVD) that allows the glass-like film to be deposited at low temperature without damaging the fiber core.

XI workshop on Resistive Plate Chambers and Related Detectors - RCP2012,

February 5-10, 2012

INFN Laboratori Nazionali di Frascati Italy

\footnotetext{
* Speaker.
} 


\section{Introduction}

The Compact Muon Solenoid (CMS) experiment [四], which is carried out at the Large Hadron Collider (LHC) in the European Center for Nuclear Research (CERN) laboratory in Geneva (Switzerland), employs Resistive Plate Chambers (RPCs) [2], []] to detect muon particles. The RPCs which are employed at CMS are composed of two bakelite plates kept $2 \mathrm{~mm}$ away by spacers. The plates are maintained at a voltage difference of about $9 \mathrm{kV}$ in order to obtain an electric field of about $4.5 \mathrm{kV} / \mathrm{mm}$ between the plates. The space between the plates is filled with an insulating gas whose ionization connected to the passage of a charged particle is used for particle detection. The gas is a mixture composed of $\mathrm{C}_{2} \mathrm{H}_{2} \mathrm{~F}_{4}$, iso- $\mathrm{C}_{4} \mathrm{H}_{10}$ and $\mathrm{SF}_{6}$ in the volume ratios 96.0/3.5/0.5 and is maintained at a fixed relative humidity of $45 \%$. A closed loop recirculation system is employed to keep the gas composition constant and uniform within the RPCs.

During the RPC operation contaminants are released in the gas mixture and this effect is responsible for an increase of the dark current until the RPCs become not usable. One of the contaminants, which has been identified and is critical due to its reactivity, is the hydrogen fluoride $(H F)$. The average $H F$ production has been estimated to be about $25 \mu \mathrm{mol} / \mathrm{h}$ which corresponds to concentrations before the filters of some part per million [ [ 9 , []] and that would quickly degrade the RPCs performance. For this reason a gas filter composed of three purifiers is installed in the system to reduce $H F$ down to $0.1 \mu \mathrm{mol} / \mathrm{h}$. Unfortunately, the filters get intoxicated after few months of use so that the $H F$ concentration must be monitored continuously to understand when the filters need to be regenerated as they are loosing their filtering capabilities.

The contaminant concentration is controlled by means of few gas sampling points, before and after each filter in the closed loop. A small amount of gas is drawn from the sampling point, the contaminants are concentrated in bottles containing a lithium hydroxyde solution whose composition is determined at fixed intervals by means of chemical (ICP-MS, INAA, etc.) and gaschromatographic analyses. This leads to a discontinuous and costly measurement procedure, while a continuous monitoring would be of great importance to optimize the RPC detecting capability.

Commercially available sensors for $H F$ detection (e.g. galvanic-type sensors which are based on $\mathrm{MnO}_{2}$ gas-diffusion electrodes [ [6]) have a measuring range of $20 \mathrm{ppm}$ which is too high for this type of measurement. More sensitive sensors such as tin dioxide and Al-doped graphene based devices [ []], [8], [9] have been proposed to detect hydrogen fluoride traces at part-per-billion concentration, but have a high cost so that the monitoring of more than 1000 RPCs can be difficult to be obtained.

Intrinsic sensors based on Plastic Optical Fibers (POF) made of polymethylmethacrylate (PMMA) [एU, प] could be an interesting alternative provided that they can be made sensitive to the $H F$. POF utilisation for sensing applications is rapidly growing [ㅁ], [3], [4] due the POF low cost and the easiness to use which is connected to their large diameter $(0.25-1 \mathrm{~mm})$ that permits to employ simple and cheap plastic connectors still maintaining a good optical coupling [15]]. Fiber optics can be used to develop intrinsic sensors thanks to several different principles (fiber grating, scintillation, light attenuation change due to pollutant absorbtion into the core, light losses due to fiber bending, etc... [[6], [1]]); POFs have, however, the advantage of having a remarkable evanescent field, which extend outside the core and can be used to develop sensors based on the propagation loss change due to the interaction of the evanescent field with the surrounding media. 
To exploit this behavior the POF cladding must be removed and the core must be coated with a substance capable of reacting with the pollutant to be measured. While the cladding removal is easy, the coating deposition may be difficult due to the PMMA sensitivity to the temperature which prevents using procedures working at more than $60-70^{\circ} \mathrm{C}$. To avoid the POF heating one can therefore take advantage of low pressure plasma ('cold plasmas') treatments. Plasma Enhanced Chemical Vapor Deposition (PECVD) processes can be used to develop very sensitive sensors [1[8], by modifying the fiber surface chemical properties either by grafting different functional groups, or by depositing thin films, whose chemical and optical properties can be changed simply by a proper selection of the experimental parameters. Moreover, plasma processes can also be used for cladding removal in order to deposit the sensitive film directly onto the fiber core without modifying its properties.

\section{Sensor design}

The critical aspect in the design of a POF sensor which exploits the evanescent field and its interaction with the surrounding media to achieve the sensitivity to the quantity of interest, is the coating selection.

The coating to be applied on the core must be capable of reacting with the sensed quantity with a reaction which alters the fiber capability of transmitting the light. In addition, to achieve the required selectivity, the coating must have a minimal reacting capability with respect to other chemical compounds, present in the surrounding environment, which could affect the light transmission. In the case of $H F$ detection, this could be easily obtained by employing a glass-like coating. The glass has a refractive index higher than the PMMA thus greatly affecting the light transmission. Furthermore it is attacked by the $F^{-}$ions deriving from the $H F$ dissociation and is almost not affected by other chemical compounds. Unfortunately, two problems arise: firstly the direct deposition of glass onto the fiber is impossible since the glass fusion temperature is much higher than the PMMA temperature, secondly the reaction with flouride ions, which leads to the formation of silicon tetrafluoride $\left(\mathrm{SiF}_{4}\right)$, is not reversible:

$$
\mathrm{SiO}_{2}+4 \mathrm{HF} \Rightarrow \mathrm{SiF}_{4}+2 \mathrm{H}_{2} \mathrm{O}
$$

This latter aspect leads to a sensor with intrinsic cumulative behavior, i.e. with an output which is proportional to the integral of the exposition to the $H F$ vapors, giving information about the filters performance. The problem of glass deposition instead can be tacked by using a coating deposited via PECVD at low temperature as explained in the next section.

\section{Fiber preparation}

All tests were performed by using a commercial step-index, highly multi-mode POF. This type of fiber is commonly used for telecommunication purposes and has negligible costs of the order of $1 \$$ per meter. The fiber has a PMMA core with a diameter of $0.98 \mathrm{~mm}$, which is surrounded by fluoropolymeric cladding which has a thickness of $0.01 \mathrm{~mm}$.

The cladding can be easily removed by using an organic solvent (such as ethylacetate) thus exposing the core onto which the coating can be deposited. 


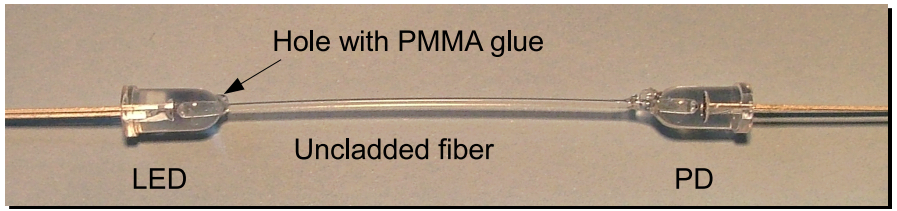

Figure 1: Sensor assembly after bonding the fiber with LED and PD.

To obtain a glass-like behavior fiber coating with a $\mathrm{SiO}_{x}$ layer, which has a structure very similar to the glass, has been performed. A real stoichiometric $\mathrm{SiO}_{2}$ layer cannot be easily obtained at low temperature, but $\mathrm{SiO}_{x}$ thin films with thicknesses of few hundred nanometers can easily be obtained by PECVD starting from organosilicon monomers.

The sensors were prepared by using a plasma fed with tetraethoxysilane (TEOS), and with a gas mixture composed of $O_{2}$ and $\mathrm{Ar}$ in different ratios. The discharge was performed at the pressure of $5 \mathrm{~Pa}$ and with an input power of $50 \mathrm{~W}$. The film thickness can be changed varying the deposition time.

The PECVD reactor consists of a stainless steel vacuum chamber with two asimmetric internal elecrodes, connected to a turbomolecular pump backed by a rotary pump. A $1 \mathrm{~kW} 13.56 \mathrm{MHz} \mathrm{RF}$ power generator connected to the cathode electrode through an impedance matching unit is used to ignite the discharge. A throttle valve and a pressure gauge permit to control the chamber pressure. Four injection lines equipped with mass flow controllers permit to use quaternary discharge gas mixtures. A separate controller is used for the film precursor vapor.

The fibers were processed onto the ground electrode at the floating temperature reached by the plasma, which was kept below $70^{\circ} \mathrm{C}$, so that avoiding to damage the PMMA core.

\section{Sensor assembly}

The basic sensor structure is composed of the coated fiber, a LED and a photodiode (PD). The fiber has a length of about $10 \mathrm{~cm}$, the LED is a common red led, which has been selected since PMMA has a stable attenuation value in the red region [ए耳] when exposed to radiations. The PD is a commercial wide-band photodiode. Both LED and PD cupolas are drilled with $1 \mathrm{~mm}$ holes; the fiber inserted into the holes and bonded to the components by using liquid PMMA. The bonding is performed on an uncladded fiber, before the PECVD deposition. The assembly is then cured in oven at $60{ }^{\circ} \mathrm{C}$ for three days and left to stabilize for other additional four days (fig. (I).

At this point teflon insulated wires are connected to LED and PD. Connections and LED and PD are then painted with an $H F$ resistant black paint and the assembly bonded to a PVC support so that the fiber are retained in a fixed position. Eventually, the painted assembly is inserted into the PECVD reactor for the SiOx film deposition. Since the reactor is not equipped with a rotating facility, the deposition is performed in two steps by reversing the fiber after half time to cover all the surface.

The described assembly procedure allows one to obtain rather cheap assemblies, but has two main drawbacks. Firstly, the initial transmission capability is strongly connected to the position of LED/PD hole with respect to cupola centers and is also strongly affected by the polishing of 


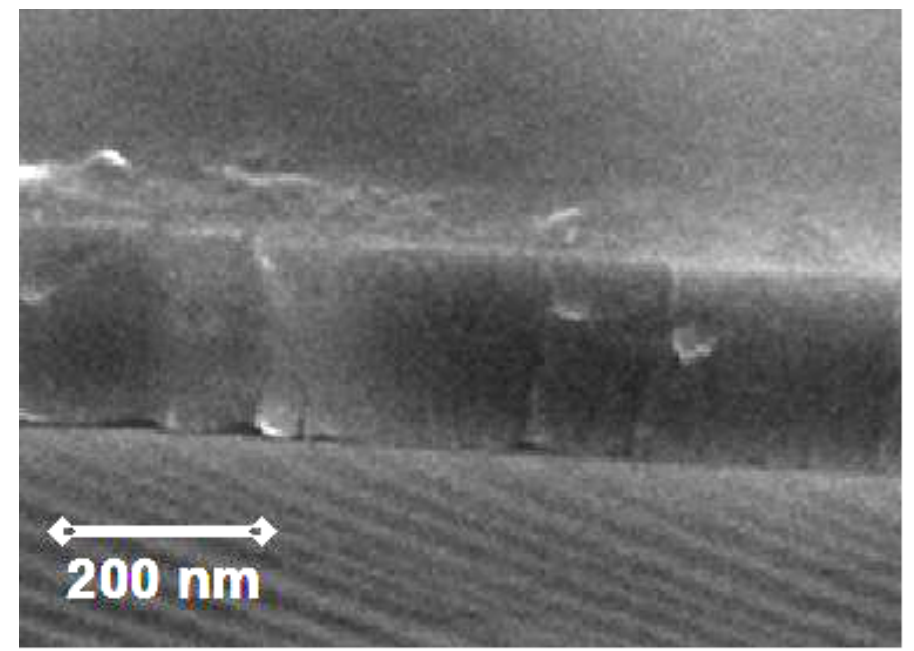

Figure 2: FESEM image of the $\mathrm{SiO}_{x}$ film cross-section. The film thickness is about $200 \mathrm{~nm}$.

the ends of the fiber. This problems may lead to rather different PD currents even in the presence of a fixed LED current and therefore the PD conditioning circuit must have a variable gain to compensate for this variability. However, if all measurement are scaled to the initial transmittance, the mounting effect can be made of minor importance. The second, more important drawback, is connected to the different expansion ratios of fiber and fiber support. This turns out in fiber stretching and/or bending, which although minimal, affect the transmission capability of about $5 \%$ for temperature changes of $5{ }^{\circ} \mathrm{C}$. This should not be a problem in the RPC use since the temperature is controlled, but a new setup is being designed to avoid this inconvenience.

\section{Experimental results}

\subsection{Fiber coating}

The fibers to be used for the tests were prepared cutting sections with length of $10 \mathrm{~cm}$. Fiber ends were polished with grinding paper. Then the cladding was removed by dipping the fiber into ethylacetate for $40 \mathrm{~s}$ and then removing the fluoropolymeric cladding with a soft paper tissue.

The the glass-like coating was deposited as described in previous section with a deposition time of $15 \mathrm{~min}$ to obtain a thickness of about $200 \mathrm{~nm}$. A Si specimen was inserted into the reactor along with the fiber to allow for a measurement of the coating thickness by means of a Field Emission Scanning Electron Microscope (FESEM). Fig. [ shows a FESEM image of the obtained coating, which shows a thickness of about $200 \mathrm{~nm}$ as expected. A mixture rich in oxygen (TEOS : $O_{2}: A r=1: 20: 10 \mathrm{sccm}$ ) was used for the deposition to obtain an inorganic coating which closely resembles the glass structure.

\subsection{Radiation effect}

Since the sensors are designed to be employed in the presence of high-energy radiations, it is important to assess the behaviors of plastic fiber, LED, and PD when exposed to such radiations. In 


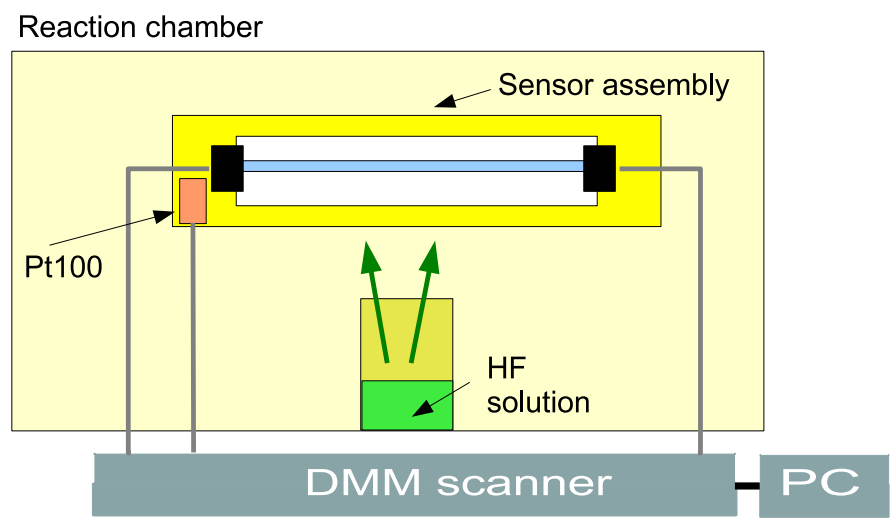

Figure 3: Block diagram of the measurement setup which comprises the sensor assembly, a resistive Pt 100 temperature sensor a DMM-scanner and a PC connected to the scanner via IEEE488 interface. The reaction chamber is made by polytetrafluoroethylene (PTFE) which is $H F$-resistant. The $H F$ vapors are generated by inserting into the reaction chamber a small PTFE bowl containing the calibrated $H F$ solution.

fact, studies are available regarding the behavior of glass fibers in the presence of radiations [23], 24], [25], but few results are available regarding the plastic fibers [प्व]. In general, high energy radiations can produce a fiber depolymerization that might reduce the fiber transparency thus impairing the sensor performance. According to the results described in [19], the expected change is of about $1 \mathrm{~dB} / \mathrm{m}$ for a PMMA fiber excited with a red wavelength and subjected to a dose of $100 \mathrm{~Gy}$. This corresponds to an amplitude change of the order of $5 \%$ on a fiber with $10 \mathrm{~cm}$ length in the same conditions, while negligible changes should be recorded for doses of the order of 1 Gy which are the values expected in the fiber installation position in several month of CMS operation. Few sensor assemblies, employing both bare and coated fibers, were installed and left in place for about six months at the Gamma Irradiation Facility (GIF) at CERN. After this period which corresponds to a dose of about $2 \mathrm{~Gy}$, no changes in the transmission ratio were recorded on the bare fibers, while a small transmission reduction, of the order of $5 \%$ was observed for the coated fibers, probably due to the chemical reaction between the fiber surface and the gas mixture.

\subsection{Exposition to $H F$}

The effect of $H F$ on the plasma deposited sensitive layer was investigated by employing the measuring setup sketched in Fig.B. The sensor assembly is arranged as described in the previous section. A Pt 100 resistive sensor is used to monitor the temperature. The setup is enclosed into a polytetrafluoroethylene (PTFE) chamber which can be completely sealed. The $H F$ vapor generation is obtained by using a small PFTE bowl containing a calibrated $H F$ solution. This way it is possible to create $H F$ vapor with different partial pressure simply by changing the concentration of the $H F$ solution. The partial pressure can be computed using the well known Antoine equation [RO] and the coefficients which can be either found at NIST site [R]] or can be computed from partial pressure experimental measurements [22].

The tests were performed by employing a certified solution containing $H F$ at a known concentration of $40 \%$ in volume. The solution was diluted to $20 \%$ in volume which corresponds to a 


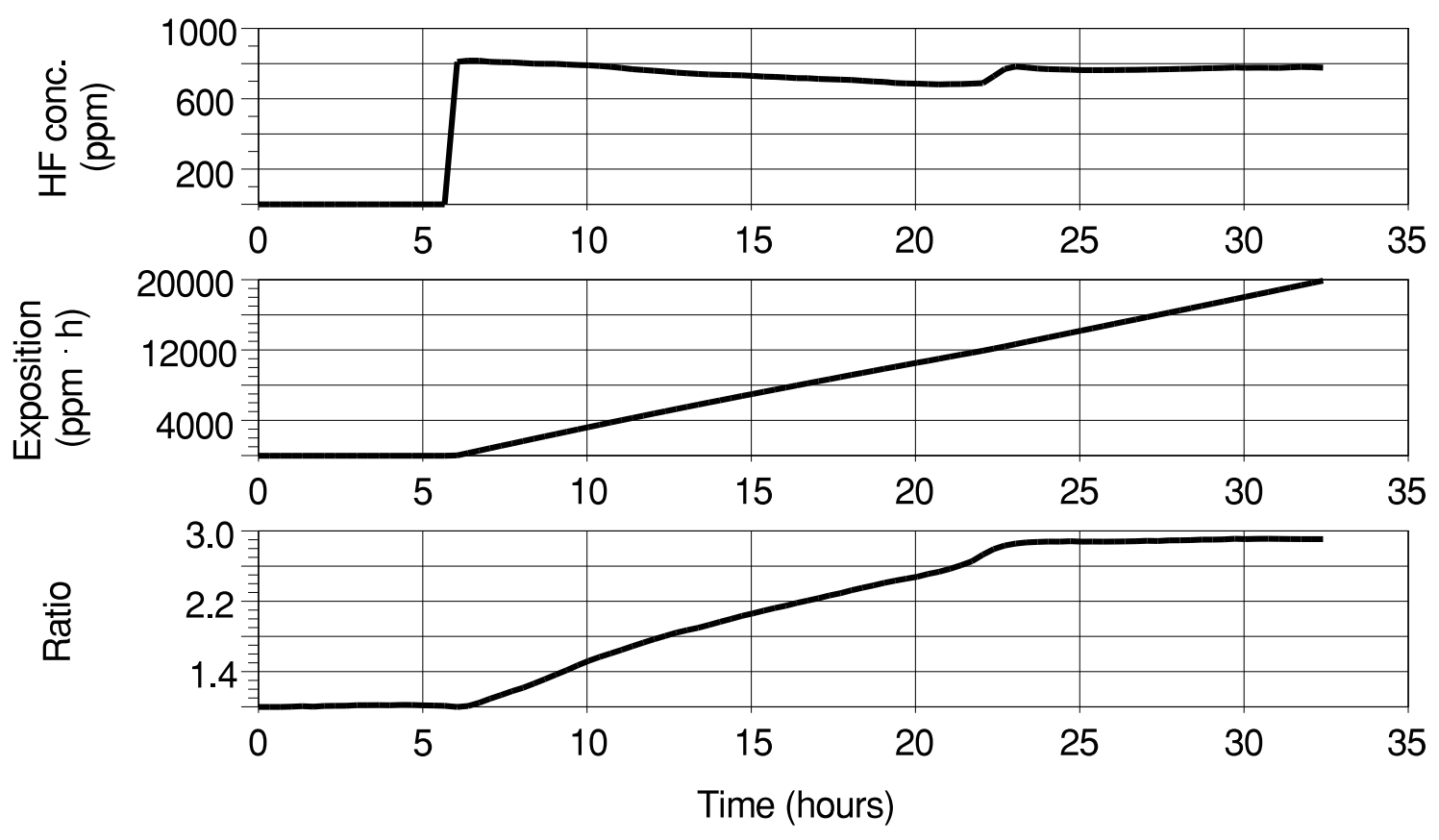

Figure 4: Coated fiber response to $H F$ exposition. Top trace: $H F$ vapor concentration computed according to the Antoine law. Middle trace: exposition computed as the integral of the vapor concentration. Bottom trace: fiber transmittance ratio normalized to its initial value. The test lasts more than two days in a not controlled environment where the temperature during the test changes in the range of $22{ }^{\circ} \mathrm{C}$ to $27^{\circ} \mathrm{C}$. The fiber has an output that follow the exposition until a saturation which appears when the transmission ratio increases of about 3 times.

partial pressure of about $700 \mathrm{~Pa}$, at the temperature of about $25^{\circ} \mathrm{C}$.

All the tests were performed by leaving the fiber into the bowl for some hours in clean air and then inserting the PTFE bowl with the $H F$ solution letting the measurement running until the fiber output remains stable.

Fig. T shows the result of a test. The top trace shows the $H F$ vapor concentration. After the initial rest interval of about 5 hours, the concentration is computed according to the Antoine law. The middle trace shows the exposition computed as the integral of the vapor concentration. The bottom trace shows the fiber transmittance ratio normalized to its initial value. The test lasted about three days in a not controlled environment where the temperature during the test changed in the range of $22{ }^{\circ} \mathrm{C}$ to $27^{\circ} \mathrm{C}$. The figure shows the first 30 hours during which the fiber has an output that follows the exposition until a saturation, which appears when the transmission ratio increases of about 3 times.

Fig. 1 shows three pictures of the fiber taken with the LED on before the deposition, after the deposition of the $\mathrm{SiO}_{x}$ layer and after the exposure to the hydrogen fluoride vapors. The pictures show how in the case of the naked fiber before the deposition (left image) the light is mostly confined inside the fiber with a small dispersion due the fiber surface irregularities which are highlighted since the cladding has been removed. The center image shows the result after the deposition of the $200 \mathrm{~nm}$ thick $\mathrm{SiO}_{x}$ layer: in this case the fiber becomes dispersive as expected since the re- 

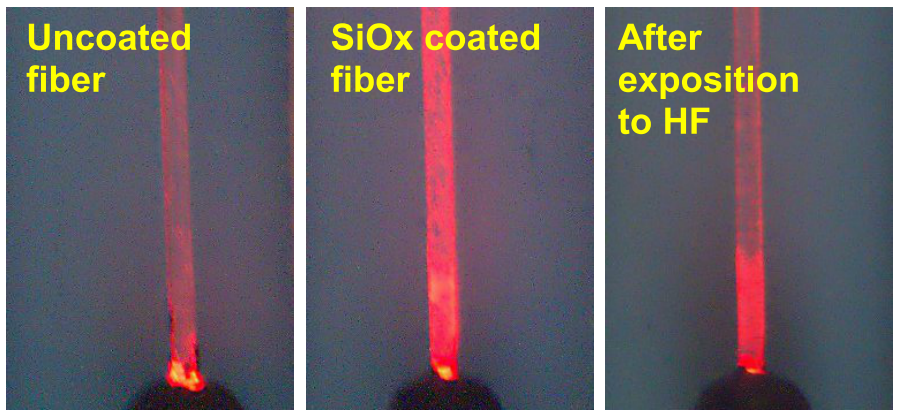

Figure 5: Effect of the $\mathrm{SiO}_{x}$ layer on the fiber light transmission capability. At left the fiber after cladding removal and before deposition: the light is mostly confined inside the fiber as expected. In the middle the fiber after the deposition of a $\mathrm{SiO}_{x}$ layer with thickness of about $200 \mathrm{~nm}$ : the refractive index of the $\mathrm{SiO}_{x}$, which is higher than the PMMA, makes the fiber dispersive. At right fiber after exposition to the $H F$ vapors. The $\mathrm{SiO}_{x}$ layer is attacked by the fluoride ions and degraded, so that the fiber is much less dispersive although still more dispersive than the uncoated fiber.
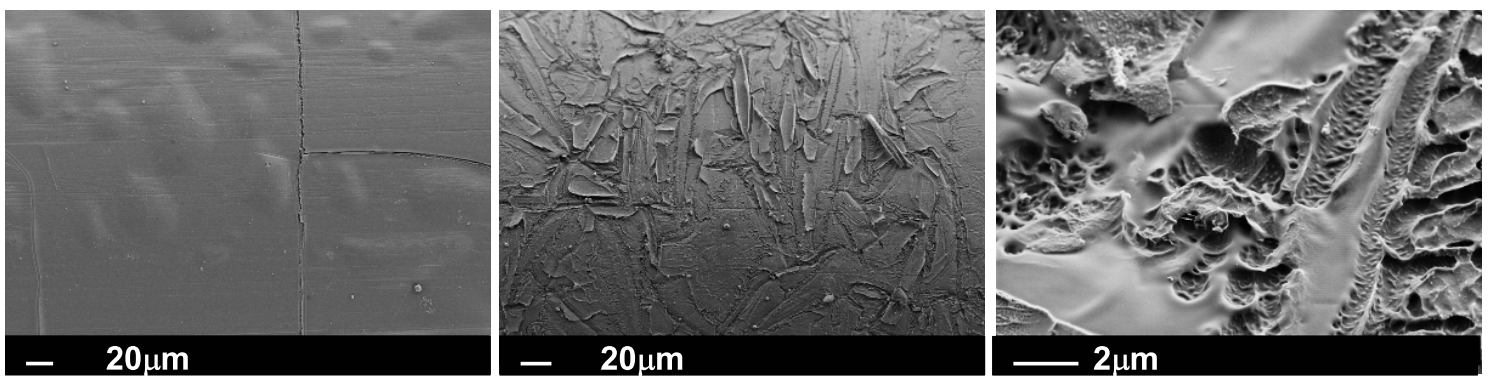

Figure 6: FESEM images of coated fiber before (left) and after (center and right) the exposition to the $H F$ vapors.

fractive index of the $\mathrm{SiO}_{x}$ film is higher than the PMMA. The right image shows the fiber after the exposition to the $H F$ vapors. The $\mathrm{Si}_{x}$ layer is almost completely corroded by the fluoride vapors so that the fiber becomes much less dispersive and this explains the received light increase.

A FESEM analysis of the fiber surface was performed; fig. 6 shows the effect of the $H F$ attack on the coating morphology. On the left the coating before the $H F$ exposition appears smooth and continuous, at center and on the right the images taken after the exposition, at different magnifications, clearly show how the coating is heavily damaged thus loosing part of its dispersing capability.

Eventually, fig. $\square$ shows a comparison between the exposure computed by integrating the $H F$ vapor concentration and the exposure $E$ estimated by the sensor output as:

$$
E=k_{f}(R-1)
$$

where $R$ is the fiber transmittance normalized to its initial value and $K_{f}$ is the transmittance sensitivity, identified starting from the experimental data, which has a value of about $7000(\mathrm{ppm} \cdot \mathrm{hour})^{-1}$. 


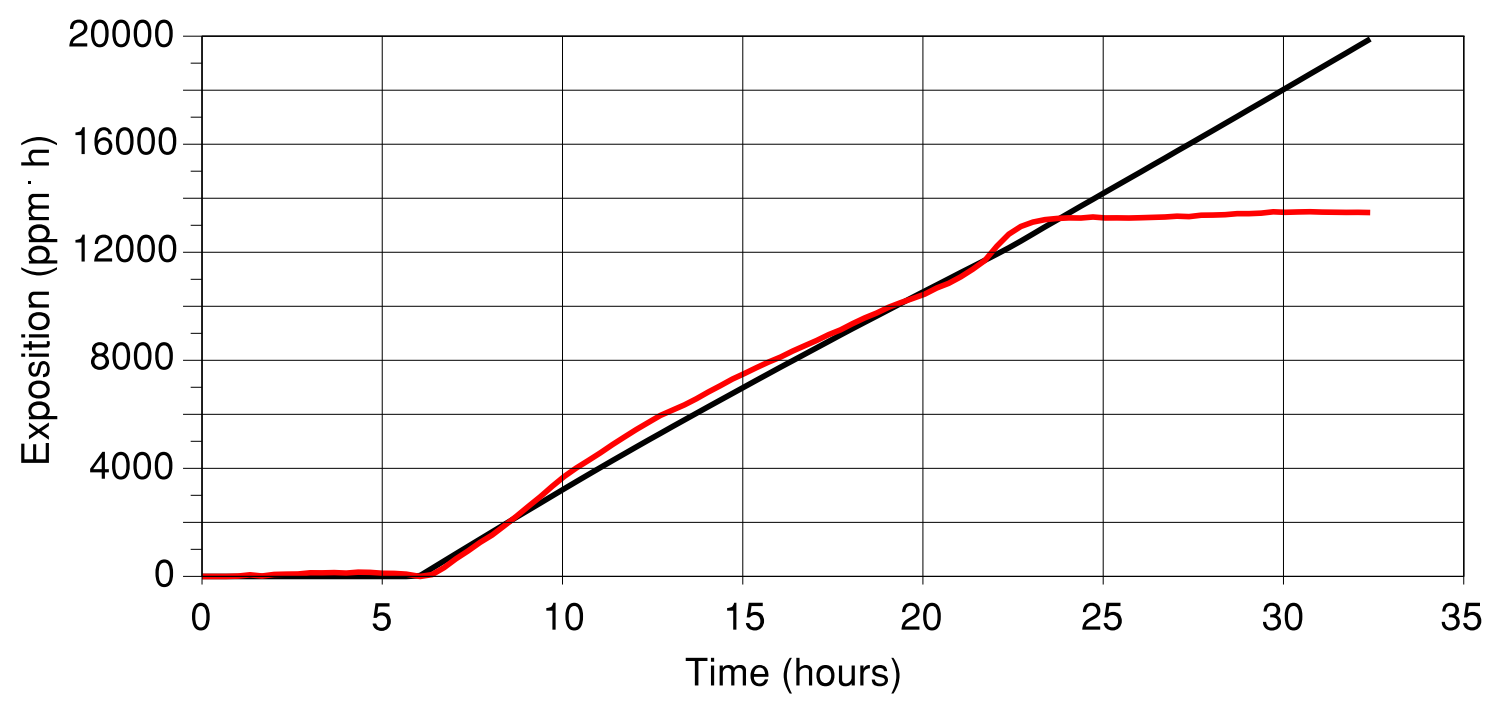

Figure 7: Comparison between the exposure as computed by integrating the $H F$ vapor concentration and the sensor response. The sensor ratio change is of about $7000(\mathrm{ppm} \cdot \text { hour })^{-1}$.

The two traces show a difference that, in the measuring range before the fiber sensor saturation, i.e. for exposures below $14000 \mathrm{ppm} \cdot \mathrm{hour}$, is below $500 \mathrm{ppm}$ i.e. less than $5 \%$ of the measuring range which is the expected transmission instability due to the non-optimal assembly mounting.

\section{Conclusions}

This paper describes a new low cost sensor which can be used for monitoring the $H F$ presence in the gas mixtures employed in the RPCs. The sensor, which is obtained by depositing a thin $\mathrm{SiO}_{x}$ layer on a commercial POF has a high selectivity with respect to the fluoride ions that are able to attack the glass-like coating. The sensor exploits a cumulative behavior thus it directly measures the total exposure to the $H F$ vapors making it suitable for estimating the gas filter intoxication level. The sensor has been tested for work in the presence of high-energy radiations and can be equipped with a wireless, battery operated controller which makes it easy its mounting within the CMS experiment facility.

The sensor still has a sensitivity with respect to the environmental conditions, which limits its accuracy and which is mainly due to the procedure followed for mounting the assembly. A new assembling structure is being designed to reduce this problem, however, the low cost of this sensor cost and its capability of working in the presence of high energy radiations make it suitable for a massive installation on all the RPCs and for a continuous gas monitoring.

\section{References}

[1] R. Adolphi et al., The CMS experiment at the CERN LHC. Published in JINST 3:S08004, 2008.

[2] R. Santonico, R. Cardarelli "Development of Resistive Plate Counters", Nucl. Instrum. Meth., 187, p. 377, 1981. 
[3] A. Colaleo et al., "First measurements of the performance of the barrel RPC system in CMS." CERN-CMS-NOTE-2008-027, Oct 2008, 15, Published in Nucl. Instrum. Meth. A, 609, p. 114, 2009.

[4] M. Abbrescia et al., "Results about HF production and bakelite analysis for the CMS Resistive Plate Chambers," Nucl. Instrum. Meth. A, 594, p. 140, 2008.

[5] L. Benussi et al., "Study of gas purifiers for the CMS RPC detector," arXiv:1012.5511 [physics.ins-det]. Presented by G. Saviano at RPC2010, Darmstadt, Germany, to appear on Nucl. Instr. and Methods, 2011.

[6] V.P. Chviruk, O.V. Linuycheva, E.M. Zaverach "Galvanic-type electrochemical sensor for determining the content of hydrohalogens in the air", Sensors and Actuators B, 92, p. 60, 2003.

[7] F.Bergera, J.B. Sancheza, O. Heintzb "Detection of hydrogen fluoride using $\mathrm{SnO}_{2}$-based gas sensors: understanding of the reactional mechanism", Sensors and Actuators B, 143, p. 152, 2009.

[8] S. Kaciulis et al. "Investigation of thin films o mixed oxides for gas-sensing applications", Surf. Interface Anal. 34, p. 672, 2002.

[9] Y. Suna, L. Chena, F. Zhangb, D. Li, H. Pana, J. Yec "First-principles studies of HF molecule adsorption on intrinsic graphene and Al-doped graphene”, Solid State Communications, 150, p. 1906, 2010.

[10] J. A. Buck "Fundamentals of optical fibers", 2nd ed., Wiley, 2004

[11] J. Zubia and J. Arrue "Plastic Optical Fibers: an introduction to their technological processes and application”, Optical Fiber Technology, 7, p. 101, 2001.

[12] U. Steiger, "Sensor properties and applications of POFs", POF'98 - 7th International Conference on Plastic Optical Fibres and Applications Conference Proceedings, Berlin, Germany, 1998.

[13] G. Durana et al. "Use of a Novel Fiber Optical Strain Sensor for Monitoring the Vertical Deflection of an Aircraft Flap”, IEEE Sensors Journal, vol. 9 no. 10, pp. 1219, 2009

[14] S. Corbellini et al. "POF sensors for gas monitoring in the presence of ionizing radiations", International Instrumentation and Measurement Technology Conference Proceedings,I2MTC 2011, Hangzhou, pp. 826, 2011.

[15] O. Ziemann et al “POF Handbook - Optical Short Range Transmission Systems”. 2nd ed., Springer, 2008

[16] B. Ruchti et al. "Development of new scintillating fiber detectors for high energy physics applications" IEEE Trans. on Nuclear Science, vol. 36, no. 1, pp. 146, 1989

[17] G. Perrone, A. Vallan, "A low-cost optical sensor for non contact vibration measurements", IEEE Trans on Instrumentation and Measurement, vol. 58, no. 7, pp. 1650, 2009.

[18] E. Angelini et al "Plastic Optic Fiber Sensor for Cumulative Measurements", Proceeding of the I2MTC 2009 - Singapore, 2009.

[19] B. Chiron "The behaviour of POF under nuclear radiation: applications for nuclear detector scintillators, data transmission \& illumination", IEE Colloquium on Plastics Materials for Optical Transmission, 5, pp. 1-8, 1989.

[20] C. Antoine , C. (1888), “Tensions des vapeurs; nouvelle relation entre les tensions et les tempè”, Comptes Rendus des Séances de l'Académie des Sciences 107: 1888.

[21] http://webbook.nist.gov/chemistry/, Sept. 2011 
[22] "Hydrofluoric Acid Properties" available at http://honeywell.com, Sept. 2011

[23] R. H. West, S. Dowling, "Measurement of long term, radiation induced losses in fibre optics using optical time domain reflectometry”, IEEE Trans. Nucl. Sci., 39, p. 418, 1992.

[24] H. Liu, D. W. Miller,J. Talnagi, "Gamma radiation resistant FabryÜPerot fiber optic sensors ”, Review of Scientific Instruments, vol. 73, p. 3112, 2002.

[25] W. H.Hardwick, A. H. Kalma, "Effects of Low-Dose-Rate Radiation on Opto-Electronic Components and the Consequences upon Fiber Optic Data Link Performance ”, IEEE Trans. Nucl. Sci., vol. 26, p. 4808, 1979.

[26] M. Tomozawa, T. Takamoriz, "Relation of Surface Structure of Glass to HF Acid Attack and Stress State", J. Amer. Ceram. Soc., vol. 62, p. 370, 1979. 